Tohoku J. exp. Med., 1983, 141, Suppl., 337-342

\title{
Analysis of Risk Factort in the Development of Diabetic Retinopathy
}

\author{
H. Kajinuma, Y. Miyake and N. Kuzuya \\ Division of Endocrinology and Metabolism, the Institute for \\ Adult Diseases, Asahi Life Foundation, Tokyo 160, Japan
}

\begin{abstract}
Kajinuma, H., Miyake, Y. and Kuzuya, N. Analysis of Risk Factors in the Development of Diabetic Retinopathy. Tohoku J. exp. Med., 1983, 141, Suppl., 337 -342 - Assumed risk factors in the development of diabetic retinopathy were examined in 401 diabetic patients who had visited our clinic at regular intervals over 5 years. These subjects showed no funduscopic abnormalities at their first visit, whereas 114 out of 401 cases $(28.4 \%$ ) developed retinopathy within 5 years while 287 cases $(71.6 \%$ ) remained unaffected. 110 cases each were selected both from the affected and unaffected groups, matching in sex, age at onset and duration, and the frequencies of various risk factors were compared. In the affected and unaffected groups, the mean maximal body weight indexes were 125 . $5 \pm 19.6 \%$ and $123.5 \pm 20.1 \%$, respectively, but the number of subjects with excessive obesity $(\geqq 130 \%)$ was greater in the former than in the latter $(p<0.05$, $x^{2}$-test). The number of patients with poor blood glucose control was greater in the former while that with good control was greater in the latter $\left(p<0.05, \chi^{2}\right.$-test). No differences were observed between the two groups for other parameters, including method of treatment. In conclusion, among the various risk factors examined, only obesity and poorer blood glucose control were significant contributors to the development of diabetic retinopathy.__ diabetic retinopathy; obesity; blood glucose control
\end{abstract}

The prevalence of diabetic retinopathy increases with the duration of diabetes ${ }^{1,2)}$. It is recognized, however, that not all diabetics of the same duration develop retinopathy, and it is felt necessary to investigate other factors than duration of diabetes to evaluate their contribution to the development of retinopathy. It has been stressed that careful control of diabetes delays the development and progression of diabetic retinopathy (Keiding et al. 1952; Miki et al. 1969; Cahill et al. 1976; Skyler 1982). But controversy still exists as to the effect of strict control of diabetes on the prevention of diabetic microvascular complications (University Group Diabetes Program 1971; Siperstein et al. 1977).

This study was designed, therefore, to assess from among many assumed risk factors, which contributes most to the development of diabetic retinopathy.

Address reprint requests to Dr. Hiroshi Kajinuma, the Division of Endocrinology and Metabolism, the Institute for Adult Diseases, Asahi Life Foundation, 1-9-14 Nishishinjuku, Shinjuku-ku, Tokyo 160, Japan. 


\section{Materials and Methods}

\section{Patients}

The subjects in this study were selected from 552 diabetic patients ( 359 males and 193 females) who visited the Diabetes Clinic of our Institute at regular intervals of every 1 to 2 months for more than 5 years during the years 1969 to 1981 . The diagnosis of diabetes was made when the patient had a fasting capillary blood glucose level (FBG) above $140 \mathrm{mg}$ / $100 \mathrm{ml}$, determined by the glucose oxidase method, or when the patient had diabetic retinopathy. Of these, 151 patients showed funduscopic abnormality at the initial visit while the remaining 401 patients did not. There was a difference in the duration of diabetes between these two groups, the former being $10.3 \pm 6.7$ years (mean \pm S.D.) while the latter was $5.0 \pm 5.8$ years. No difference was observed in the age distributions at onset or at initial visit.

114 out of 401 cases $(28.4 \%$ ) developed retinopathy within 5 years while 287 cases ( 71 . $6 \%$ ) remained unaffected. 110 cases each were selected from the affected and unaffected groups, matching in sex, age at onset, and duration of diabetes. They consisted of 73 males and 37 females each; the ages at onset were $45.9 \pm 11.4$ years in the affected group and 46 . $6 \pm 10.5$ years in the unaffected group; the duration being $5.8 \pm 5.8$ and $5.7 \pm 6.0$ years, respectively.

\section{Factors examined}

Comparisons were made between the two groups on the following items: maximal and initial body weight index (BWI), weight control, initial FBG, blood glucose control, blood pressure, serum cholesterol, triglyceride, creatinine, and blood urea nitrogen.

\section{Criteria of control of diabetes}

Degree of control of diabetes was assessed by the course of FBG levels which were determined by a glucose oxidase method. Blood glucose control was defined as "good" when more than $80 \%$ of FBG remained below $120 \mathrm{mg} / 100 \mathrm{ml}$. "Poor" control was assigned when more than $50 \%$ of FBG was higher than $150 \mathrm{mg} / 100 \mathrm{ml}$. "Fair" control was defined as lying between good and poor controls.

Weight control was defined as "excellent" when ideal body weight (IBW) was maintained throughout the whole period, "good" when IBW was attained within 5 years though the patient had been obese at the initial visit, as "fair" when BWI of 110 to $120 \%$ was maintained throughout the 5 year period, and as "poor" when WBI of more than $120 \%$ was maintained over the 5 years.

IBW and BWI were calculated according to the following equations :

$$
\mathrm{BWI}=\frac{\begin{array}{l}
\mathrm{IBW}(\mathrm{kg})=[\text { height }(\mathrm{cm})-100] \times 0.9 \\
\text { body weight at the time of study }
\end{array}}{\mathrm{IBW}} \times 100
$$

\section{Ophthalmologic examination}

Ocular funduscopic examination was performed using ophthalmoscopy at least once a year, and the severity of the finding was classified accomding to Scott's criteria (1953).

\section{Statistical analysis}

Multiple logistic analysis was performed using the 10 variables described above at the initial visit, to estimate the risk of diabetic retinopathy. Whether the difference in the distribution pattern was significant was calculated using the $\boldsymbol{\chi}^{2}$-test. 


\section{Results}

Ten variables were used to calculate the multiple logistic function using the data obtained from all 401 patients who had no retinopathy at initial visit, but no variable was found to be a significant risk factor of diabetic retinopathy.

Among 110 affected patients, 80 developed Scott I retinopathy, 29 Scott II and 1 Scott IIIa. The number of patients undergoing mode of treatment in the affected and unaffected groups were: diet, 23 and 23 ; oral hypoglycemic agents, 58 and 59 ; and insulin, 29 and 28 ; respectively.

Fig. 1 illustrates the distributions of maximal BWI, BWI at initial visit, and weight control in the retinopathy-developing and control group matched for sex, age at onset and duration. In the control group, the highest incidence was observed in the group showing a maximal BWI of 110 to $130 \%$, while in the affected group the incidence increased in proportion to the maximal BWI, and the highest incidence was observed in the group showing a maximal BWI of more than $130 \%$. There was a significant difference in the distribution pattern between these two groups. The same result was obtained when subjects were limited to those with severer retinopathy (Scott II and above). On the other hand, no differences were observed in the distributions of BWI at initial visit or

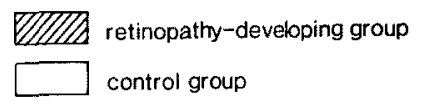

Maximal body weight index

Body weight index

Weight control

at the initial visit
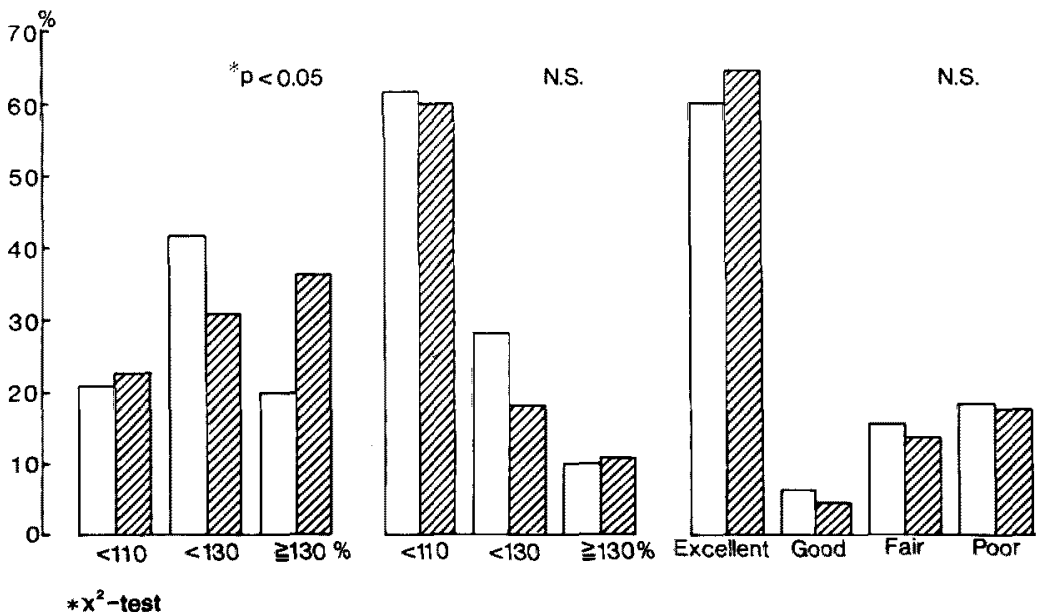

$* \mathrm{x}^{2}$-test

Fig. 1. Comparison of the distribution of maximal body weight index, body weight index at initial visit and weight control between the retinopathy-developing and the sex, age and duration-matched control groups. All cases of retinopathy-developing patients are included. 

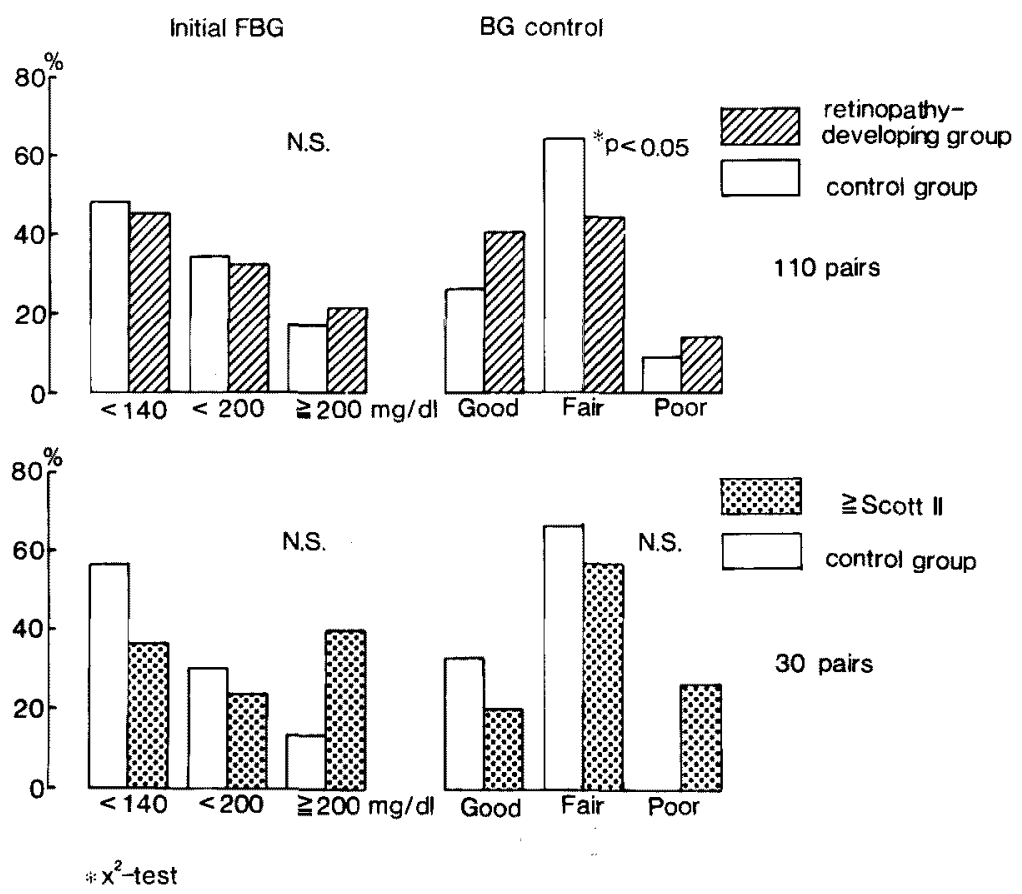

30 pairs

Fig. 2. Comparison of the distribution of initial fasting blood glucose and degree of control between the retinopathy-developing and the sex, age and duration-matched control groups. All cases of retinopathy-developing groups are shown above, and patients with retinopathy of Scott II and more are shown below.

of weight control, as shown in Fig. 1.

Fig. 2 shows the distributions of initial FBG and degree of blood glucose control in the retinopathy-developing and control groups. There was no difference between the two groups in the distribution of initial FBG. However, the incidence of fair control was considerably higher in the control group than in the affected group and the distribution pattern for blood glucose control differed between the two groups. The same tendency was observed when subjects were limited to those with severer retinopathy, but the difference was not significant.

No differences were observed between the two groups in the distribution patterns for other items : blood pressure, serum cholesterol, triglyceride, creatinine or blood urea nitrogen.

\section{Discussion}

No one single factor is responsible for the development of diabetic retinopathy. From the standpoint of patient care, it is necessary to know which of these many factors is (are) most significant in the development of diabetic retinopathy, and for this purpose we attempted to compare the incidence of assumed risk factors between the retinopathy positive and negative groups. However, the patients were heterogenous in terms of sex, age at onset and duration, and because 
all these three factors affect the course of diabetic microvascular complications (Bradley and Ramos 1973), it seemed inappropriate to study the incidence of retinopathy using these heterogenous groups.

In order to make the comparison easier, we selected patients who did not show any funduscopic abnormality at the first visit. Of these, 114 patients developed retinopathy during the course of the 5 year observation period while the remaining 287 cases did not. 110 cases each were selected from both groups, matched for sex, age at onset and duration of diabetes, and the incidence of various risk factors were compared between these two groups. Thus, this method had the advantage of selecting patients who were homogenous as far as possible in respect to these three variables.

This study suggested that past history of obesity and blood glucose control during the course of the 5 year follow-up influenced the occurrence of diabetic retinopathy.

Obesity has by far the greatest influence on the onset of diabetes (Marks et al. 1973). This study showed that it also had a great influence on the development of diabetic retinopathy. It may be argued that in obese diabetics, insulin action on the microvasculature of the retina is diminished as it is on the fat cells ${ }^{10)}$, thus leading to the development of specific diabetic retinal changes.

It is interesting to note that a past history of extreme obesity, rather than weight control after the initiation of treatment at our clinic, influenced the devenopment of retinopathy. This may indicate that the past history of extreme obesity overshadowed the effect of weight control during the relatively short 5 year period. It may be necessary to carry out further observations for a longer period to characterize the effect of weight control on the development of retinopathy.

The degree of blood glucose control affected the occurrence of diabetic retinopathy, in keeping with earlier reports (Keiding et al. 1952 ; Miki et al. 1969 ; Cahill et al. 1976; Skyler 1982). Bodansky et al (1982). reported that not only current poor diabetic control, but also hypertension and previous treatment with once-daily insulin regimen served as risk factors for proliferative retinopathy. In our patients hypertension and mode of treatment did not affect the development of diabetic retinopathy. This discrepancy was obviously due to the differences in patient characteristics; their patients were all insulin-dependent and the duration of diabetes was far longer in their study. Further observation is needed to clarify the effects of these factors, using the new method adopted in this study.

\section{Acknowledgments}

The authors wish to thank Miss Takako Yasunishi and Mrs. Miharu Kawasaki for their assistance. They are also grateful to the clinical, nursing and laboratory staff of the Institute for Adult Diseases, Asahi Life Foundation, Tokyo. 


\section{References}

1) Bodansky, H.J., Cudworth, A.G., Drury, P.L. \& Kohner, E.M. (1982) Risk factors associated with severe proliferative retinopathy in insulin-dependent diabetes mellitus. Diabetes Care, 5, 97-100.

2) Bradley, R.F. \& Ramos, E. (1973) They eyes and diabetes. In: Joslin's Diabetes Mellitus, edited by A. Marble, P. White, R.F. Bradley \& L.P. Krall, Lea \& Febiger, Philadelphia, pp. 478-525.

3) Cahill, G.F., Jr., Etzwiler, D.D. \& Freinkel, N. (1976) Blood glucose controm in diabetes. Diabetes, 25, 237-239.

4) Davidson, M.B. (1972) Effect of obesity on insulin sensitivity of human adipose tissue. Diabetes, 21, 6-12.

5) Keiding, N.R., Root, H.F. \& Marble, A. (1952) Importance of control of diabetes in prevention of vascular complications. J. Amer. med. Ass., 150, 964-969.

6) Marks, H.H., Krall, L.P. \& White, P. (1973) Epidemiology and detection of diabtes. In: Joslin's Diabetes Mellitus, edited by A. Marble, P. White, R.F. Bradley \& L.P. Krall, Lea \& Febiger, Philadelphia, pp. 10-34.

7) Miki, E., Fukuda, M., Kuzuya, T., Kosaka, K. \& Nakao, K. (1969) Relation of the course of retinopathy to control of diabetes, age, and therapeutic agents in diabetic Japanese patients. Diabetes, 18, 773-780.

8) Scott, G.I. (1953) Ocular complications of diabetes mellitus. Brit. J. Ophthalmol., 37, 705-715.

9) Siperstein, M.D., Foster, D.W., Knowles, H.C., Jr., Levine, R., Madison, L.L. \& Roth, J. (1977) Control of blood glucose and diabetic vascular disease. New Engl. J. Med., 296, 1060-1063.

10) Skyler, J.S. (1982) Diabetic retinopathy. J. Amer. med. Ass., 247, 3246-3247.

11) University Group Diabetes Program (1971) Effects of hypoglycenic agemts on the vaseular complications in patients with adult-onset diabetes. III. Clinical implications of UGDP results. J. Amer. med. Ass., 218, 1400-1410. 